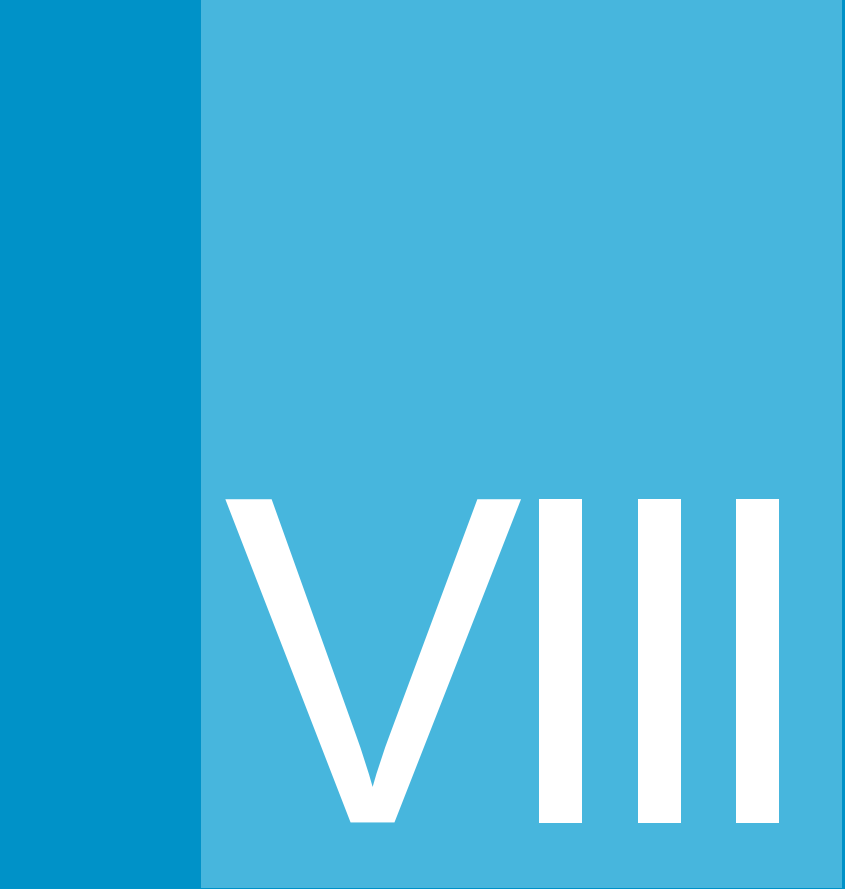

\title{
COMPETENCIA LECTORA: FACTORES CLAVE DE LOS PAÍSES DESTACADOS EN PISA Y POSTURA DE LA EDUCACIÓN ADVENTISTA
}

\section{Reading competence: Key factors of the leading countries in PISA and posture of Adventist education \\ Universidad Peruana Unión}

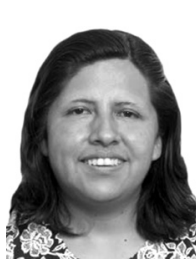

\section{Belinda Maquera Chambi}

Licenciada en Lingüística y Literatura por la Universidad Peruana Unión. Licenciada en Educación Primaria por la Universidad Peruana Unión. Magíster en Investigación y Docencia Universitaria por la Universidad Peruana Unión. Doctorando en Currículo y Docencia por la Universidad Peruana Unión. Docente Principal de la Universidad Peruana Unión en la cátedra de lingüística y literatura. Ha participado como Especialista de Comunicación en el Programa Nacional de Formación y Capacitación Permanente (PRONAFCAP) conducido por la Universidad Peruana Unión, en convenio con el Ministerio de Educación del Perú. Ha sido ponente a nivel nacional e internacional en congresos de educación e investigación. 


\section{Resumen}

El presente trabajo constituye una revisión teórica y busca identificar los factores clave presentes en el éxito alcanzado en competencia lectora por parte de los países destacados en PISA, además da a conocer aspectos fundamentales del modelo educativo adventista y su posición frente a la lectura. Este estudio se realizó en base a informes de los resultados de las evaluaciones PISA: 2000 a 2012, artículos de analistas educativos, textos del Espíritu de Profecía, así como textos de Pedagogía Adventista. Los datos fueron autenticados sometiéndolos a la crítica externa e interna. Se concluye que los factores clave para lograr el éxito en la competencia lectora son entre otros: Presupuesto conveniente, equipamiento e infraestructura adecuados, calidad de los maestros, incremento de horas en asignaturas básicas, textos escolares apropiados, aplicación de procesos cognitivos, enfoque sociolingüístico para enseñar comunicación y expectativas de los padres.

Palabras clave: Evaluación PISA, modelos educativos destacados, factores clave para la competencia lectora.

\section{Abstract}

This paper is a theoretical review and seeks to identify the key factors in the success in reading literacy by leading countries in PISA also discloses fundamental aspects of Adventist educational model and its position on reading. This study was based on reports of the results of the PISA assessments: 2000-2012, analysts educational articles, texts of the Spirit of Prophecy, as well as texts of Adventist Education. Data were authenticated by subjecting them to external and internal criticism. It is concluded that the key factors for success in reading literacy among others: convenient budget, equipment and adequate infrastructure, quality of teachers, increase in hours in core subjects, appropriate textbooks, application of cognitive, sociolinguistic approach to teach communication and parental expectations.

Keywords: PISA evaluation, prominent educational models, key factors for reading literacy. 


\section{Introducción}

Una de las competencias básicas que debe dominar el ciudadano del siglo XXI es la competencia lectora; sin embargo, Perú, en relación a tal competencia, entre otras básicas, ocupa los últimos lugares en las evaluaciones internacionales. El propósito de la presente investigación es identificar los factores clave que inciden para que los países destacados logren tal nivel de calidad en la competencia lectora de sus estudiantes del nivel secundario. Así, se pretende dar respuesta a las siguientes interrogantes: ¿En qué consiste la evaluación de la competencia lectora según PISA?, ¿cuáles son las características de los modelos educativos que destacan en la competencia lectora a nivel mundial?, ¿cuáles son las características del modelo educativo adventista y su posición frente a la competencia lectora? y ¿cuáles son los factores clave para mejorar la calidad de la competencia lectora en las instituciones educativas?

\section{Evaluación de la competencia lectora según PISA}

El Programa para la Evaluación Internacional de Alumnos (PISA) es aplicado por la Organización para la Cooperación y el Desarrollo Económico (OCDE). Esta evaluación provee información acerca del nivel de logro de los estudiantes en capacidades y habilidades fundamentales, así como de los factores escolares y extraescolares que se asocian a dichos logros. El Perú ha participado los años 2001, 2009, 2012 y ha comprometido su participación en el 2015; si bien los estudiantes peruanos mostraron resultados bajos en comparación a otros países de América Latina, se reporta un progreso sostenido en los últimos 11 años; es decir, que entre 2001 y 2012 se ha incrementado el promedio peruano de 327 a 384 puntos. En la última evaluación 2012 en relación a la de 2009 se ha subido 14 puntos, el más alto progreso entre los países de América Latina participantes en PISA (UMC, 2013, párr. 6).

Las evaluaciones PISA son aplicadas cada tres años para examinar el rendimiento de alumnos de 15 años en lectura, matemáticas y ciencias. Además estas evaluaciones permiten estudiar la motivación de los estudiantes por aprender, la concepción que tienen sobre sí mismos y sus estrategias de aprendizaje. En el presente estudio se considerarán únicamente los resultados obtenidos en competencia lectora y los modelos educativos de los países destacados en dicha competencia: Finlandia, Corea del Sur y Shangái (China). 
Tabla 1

Países destacados en competencia lectora según las evaluaciones PISA

\begin{tabular}{|c|c|c|c|c|c|c|c|c|c|c|}
\hline \multirow{2}{*}{$\begin{array}{l}\text { 离 } \\
\text { แ్ }\end{array}$} & \multicolumn{2}{|c|}{$\begin{array}{l}\text { Evaluación } \\
2000\end{array}$} & \multicolumn{2}{|c|}{ Evaluación 2003} & \multicolumn{2}{|c|}{ Evaluación 2006} & \multicolumn{2}{|c|}{ Evaluación 2009} & \multicolumn{2}{|c|}{$\begin{array}{l}\text { Evaluación } \\
\quad 2012\end{array}$} \\
\hline & País & Pt. & País & Pt. & País & Pt. & País & Pt. & País & Pt. \\
\hline $1 .^{\circ}$ & Finlandia & 546 & Finlandia & 543 & Corea del Sur & 556 & $\begin{array}{l}\text { Shangái } \\
\text { (China) }\end{array}$ & 556 & $\begin{array}{l}\text { Shangái } \\
\text { (China) }\end{array}$ & 570 \\
\hline $2 .^{\circ}$ & Canadá & 534 & Corea del Sur & 534 & Finlandia & 547 & $\begin{array}{l}\text { Corea del } \\
\text { Sur }\end{array}$ & 539 & $\begin{array}{l}\text { Hong } \\
\text { Kong } \\
\text { (China) }\end{array}$ & 545 \\
\hline $3 .^{\circ}$ & $\begin{array}{l}\text { Nueva } \\
\text { Zelanda }\end{array}$ & 529 & Canadá & 528 & $\begin{array}{l}\text { Hong Kong } \\
\text { (China) }\end{array}$ & 536 & Finlandia & 536 & Singapur & 542 \\
\hline $4 .^{\circ}$ & Australia & 528 & Australia & 525 & Canadá & 527 & $\begin{array}{l}\text { Hong } \\
\text { Kong } \\
\text { (China) }\end{array}$ & 533 & Japón & 538 \\
\hline $5 .^{\circ}$ & Irlanda & 527 & Liechtenstein & 525 & Nueva Zelanda & 521 & Singapur & 526 & $\begin{array}{l}\text { Corea del } \\
\text { Sur }\end{array}$ & 536 \\
\hline \multirow[t]{2}{*}{$6 .^{\circ}$} & Corea & 525 & Nueva Zelanda & 522 & Irlanda & 517 & Canadá & 524 & Finlandia & 524 \\
\hline & \multicolumn{2}{|c|}{$\begin{array}{l}\text { Media de la } \\
\text { OCDE: } 500\end{array}$} & \multicolumn{2}{|l|}{$\begin{array}{l}\text { Media de la } \\
\text { OCDE:494 }\end{array}$} & \multicolumn{2}{|c|}{$\begin{array}{l}\text { Media de la OCDE: } \\
\qquad 500\end{array}$} & \multicolumn{2}{|c|}{$\begin{array}{l}\text { Media de la } \\
\text { OCDE: } 493\end{array}$} & \multicolumn{2}{|c|}{$\begin{array}{l}\text { Media de la } \\
\text { OCDE: } 496\end{array}$} \\
\hline
\end{tabular}

Fuente elaborada en base a los informes de la OCDE de los resultados de las evaluaciones PISA $2000,2003,2006,2009$ y 2012. Pt = puntos.

Según el comité de expertos de la $\operatorname{OCDE}(\mathrm{s} / \mathrm{f}, \mathrm{p} .8)$ la competencia lectora es la capacidad de un individuo para comprender, utilizar y reflexionar sobre textos escritos, con el propósito de alcanzar sus objetivos personales, desarrollar su conocimiento y sus capacidades, además de participar en la sociedad. Tiene tres dimensiones: el tipo de reactivo de lectura, la forma y estructura del material de lectura, y el uso para el cual se creó el texto.

\section{Características de los modelos educativos que destacan en la competencia lectora a nivel mundial}

Luego de haber identificado los países que obtienen mejores resultados en las evaluaciones PISA, vamos a caracterizar los modelos educativos más resaltantes, basándonos en el artículo de Torres (2013, pp. 27 - 29).

\section{Modelo educativo de China}

- Modelo educativo selectivo, estresante, tremendamente competitivo, sostenido por un estilo parental autoritario y exigente con el estudio. 
- La educación obligatoria llega hasta los 15 años de edad y se inicia desde los 2 y 3 años. Los niños preescolares son preparados en nociones básicas de lectura, escritura, cálculo e inglés, para la exigente vida escolar futura.

- Se enfatiza la memoria y se aplica abundantes pruebas estandarizadas.

- Los estudiantes viven presionados por aprobar las pruebas estandarizadas y ubicarse en los primeros lugares, pues de ello depende sus futuras posibilidades de estudio, trabajo y estatus social.

- Dicha presión tiene como consecuencia altos niveles de depresión y estrés, generando entre los estudiantes altas tasas de suicidio.

- El examen de ingreso a la universidad es exigente y selectivo; así China ha logrado colocarse como líder mundial en producción de doctores.

- La calidad de la educación varía bastante entre zonas rurales y urbanas.

- La última reforma educativa china busca un nuevo marco de evaluación escolar que reduce el uso de las pruebas estandarizadas y recurre a otros indicadores.

- Shangái - capital económica de China, con más de 20 millones de habitantes-, ocupa el primer lugar en competencia lectora en 2009 y 2012. Más del $80 \%$ de sus estudiantes toma clases particulares extra, además de dedicar entre 3 a 5 horas diarias para realizar sus tareas.

\section{Modelo educativo de Corea del Sur}

- Las últimas seis décadas ha logrado un avance extraordinario en educación con políticas consistentes y perseverancia, pero a un alto costo social: calendario de estudios extendido; 50 horas semanales entre clases regulares y refuerzo escolar.

- Por la alta sobrecarga de estudios el agotamiento y déficit de sueño afectan a millones de estudiantes. La tasa de suicidio y depresión adolescente de Corea del Sur es una de las más altas entre los países con poder económico.

- El punto culminante de la tensión escolar está alrededor del tercer año de la educación secundaria, que es cuando los estudiantes dan el examen de ingreso a la universidad.

- Los profesores son bien remunerados (mejor que en Finlandia) pero trabajan bajo mucho estrés y con grupos numerosos.

- Un sistema escolar paralelo opera y crece al lado del sistema escolar público; academias privadas que ayudan a hacer las tareas, preparan para las pruebas, refuerzan contenidos escolares o avanzan más del aprendizaje en aula. 


\section{Modelo educativo de Finlandia}

Además de Torres (2013, pp. 27 - 29) para caracterizar este modelo, también nos basamos en el artículo de Hernández (2013, pp. 6 - 13).

- Finlandia, un país pequeño (poco más de 5 millones y medio de habitantes) ubicado al norte de Europa, destaca en los rankings mundiales de educación y en otros ámbitos como: desarrollo humano, calidad de vida, equidad de género, cuidado infantil, condiciones para la maternidad, acceso a Internet (banda ancha), transparencia, baja corrupción, alta competitividad internacional, entre otros.

- Sistema descentralizado y flexible: La política educativa es responsabilidad del Ministerio de Educación y de la Dirección Nacional de Educación, quien elabora el currículo nacional.

- Cuerpo docente: Muchos especialistas lo destacan como el quid del éxito finlandés, en él destacan dos cualidades: formación docente de excelencia, pues se necesita una calificación de nueve sobre 10. La segunda cualidad es alta estima social, el sueldo en promedio (datos del 2010) es de 37000 455 dólares anuales, el docente es un referente educativo que asume un cierto liderazgo entre la comunidad.

- El $90 \%$ del sistema escolar es público y gratuito, desde inicial hasta la universidad, incluidos costos de transporte, alimentación y refuerzo escolar. Incluso las pocas instituciones privadas están bajo la supervisión pública y son financiadas por el Estado. Finlandia destina 6,8\% del PBI y está por encima del promedio OCDE que es de $6 \%$.

- Se promueve la cooperación y la no competencia (en el sentido de evitar el concurso) entre alumnos, profesores e instituciones y se da prioridad a la equidad sobre la excelencia.

- El bienestar emocional de los estudiantes -aspecto ignorado en las evaluaciones de PISA- es tanto o más importante que su rendimiento escolar. La disciplina se mantiene en un nivel alto, se atiende la diversidad de los estudiantes designando dos profesores por aula.

- La escolaridad empieza entre los seis y los siete años; antes se prioriza el juego. Al año siguiente inician la educación primaria. Son obligatorios nueve años de educación (de los siete a los 16).

- La enseñanza se realiza en grupos pequeños, no más de 20 alumnos y en un ambiente relajado.

- Se desincentiva la memorización y se estimula el pensamiento, la creatividad y la autonomía de alumnos y profesores. 
- El año escolar es uno de los más cortos de los países de la OCDE, la jornada escolar diaria se organiza cuidando de respetar los ritmos biológicos del niño y evitar todo cansancio inútil. Las sesiones de 45 minutos se entrecruzan con periodos de descanso de 15 minutos. Los deberes en casa se reducen al mínimo sobre todo en inicial.

- No se aplican pruebas estandarizadas. Los alumnos son evaluados a partir de los nueve años con una escala que prescinde de cifras, desde el 6to grado aparece una escala que va del 4 al 10. Los maestros son evaluados por otros colegas más experimentados y reciben de ellos refuerzo; asimismo son evaluados por los estudiantes y por sus familias, mediante cuestionarios que pueden responderse a través de Internet.

- No existe "pago por mérito". No hay supervisores ni inspectores, el $90 \%$ de la sociedad finlandesa confía en el profesionalismo de sus docentes.

- Existe coordinación entre la familia, la escuela y las estructuras socioculturales de apoyo educativo. En este país domina la tradición luterana, de ahí el sentido de responsabilidad y disciplina sobre la propia vida. Finlandia promueve la necesidad de la lectura personal de la Biblia para el desarrollo de los valores y la espiritualidad (Vilela, 2010, párr. 13). La familia finlandesa se considera la primera responsable de la educación de sus hijos, los padres y madres son ávidos lectores y acuden con sus hijos a las bibliotecas con frecuencia. Los medios de comunicación como estructura social de apoyo educativo difunden todos sus programas subtitulados para promover la lectura natural.

- Lo negativo, es que empiezan a percibirse síntomas de fatiga entre la población infantil y adolescente, atribuidos al excesivo tiempo dedicado a hobbies y al mayor estrés de los adultos en la familia; antes que al sistema escolar.

\section{Características del modelo educativo adventista y su posición frente a la} competencia lectora

- $\quad$ El primer deber del estudiante y del maestro hacia Dios y sus semejantes es el desarrollo de sí mismos (Cadwallader, 2010, p. 52).

- La verdadera educación es más que estudiar, más que preparar al estudiante para su desempeño en esta vida; comprende todo el ser, toda la vida; es el desarrollo equilibrado de las facultades físicas, mentales, espirituales y [sociales], pues forma al estudiante para servir en la tierra y en el cielo (White, 1958, p. 11).

- Las fuentes de la verdadera educación son: la revelación de la Biblia, 
las experiencias del trabajo y de la vida, y la investigación científica (Cadwallader, 2010, p. 58).

- La Biblia debiera tener el primer lugar en todo sistema educativo (White, 1923, p. 542). Es el libro educativo más completo del mundo y el más efectivo para la formación intelectual (Ibídem, 2010, p. 415).

- La educación es una tarea noble y elevada (Ibídem, p. 54).

- La indolencia y la negligencia al adquirir la educación, es un pecado (White, 1957, p. 183).

- El verdadero conocimiento y desarrollo se originan en el conocimiento de Dios, toda investigación para llegar a la verdad pone en contacto a la mente humana con la inteligencia divina (White, 1958, p. 12).

- La educación de las masas y el mejoramiento de la sociedad, empieza en la época temprana de la vida, mediante los hábitos formados en la infancia y la juventud (White, 1957, p. 231).

- Durante los primeros ocho o diez años de la vida del niño, el campo o el jardín constituyen la mejor aula, la madre, la mejor maestra y la naturaleza el mejor libro de texto. (White, 1974, p. 280).

- El docente debe poseer cualidades como: vocación; intelecto superior; madurez; experiencia; capacidad administrativa; conocimiento profundo de las ciencias; habilidades mentales superiores; carácter noble y equilibrado; cultura amplia; dominio de las materias que enseña; capacidad de habla y lectura oral; dominio de su lengua materna; comprensión de la fisiología; saber tratar con la mente humana; equilibrio físico, mental y moral; amar a los estudiantes, eficiencia; modales refinados; modelo y ejemplo de lo que desea que lleguen a ser sus alumnos; responsabilidad; dominio propio; juicio; principios éticos sólidos; régimen adecuado en la alimentación, el trabajo, el vestir y la recreación. En caso de ser director: intuición aguda, dejarse guiar por el Espíritu de Dios, habilidad para entender el carácter, capacidad de dirigir, tacto y sabiduría. (Cadwallader, 2010, pp. $125-130)$.

- La carga laboral del docente no debe sobrepasar su fuerza física ni su energía nerviosa, a fin de que trate bien a los alumnos y pueda mantener su salud. (Ibídem, p. 145).

- Antes de pretender adquirir conocimientos superiores, el docente debe asegurarse que los alumnos comprenden a cabalidad las reglas de la gramática y que han aprendido a leer, escribir y poseen una correcta ortografía (White, 1957, p.12).

- El progreso será notorio cuando los alumnos dominen la elocución, la 
lectura, la escritura y la ortografía (White, 2008 a, p. 114).

- El estudio del idioma es un saber fundamental en toda escuela y se debe enseñar su uso correcto al hablar, leer y escribir; para poder ejercer una influencia mucho mayor en los oyentes (Ibídem).

- La educación manual merece más atención de la que se le ha prestado, la escuela debe propiciar el desarrollo físico y la capacitación industrial (White, 1958, p. 120).

- No se debe abarcar mucho estudio. La salud debe protegerse de modo tan sagrado como el carácter (White, 2008, p. 179).

- La tarea más extraordinaria y fructífera de la verdadera educación es la edificación del carácter y la formación de la personalidad (White, s/f, p. 5). Es urgente que los jóvenes formen hábitos de dominio propio, aplicación y confianza propia, de respeto a los superiores y de reverencia a Dios; así aprenderán a honrar a su Creador y ser una bendición para sus semejantes (White, 1923, p. 64).

Factores clave para mejorar la calidad de la competencia lectora en las instituciones educativas

Frente a los resultados obtenidos por nuestro país en las evaluaciones PISA lo que importa es saber cómo mejorar esos resultados; los factores clave, precisamente, constituyen el cómo y se desprenden de los modelos educativos presentados, entre tales factores están:

\section{Presupuesto conveniente}

La educación requiere inversión. Aunque la relación entre inversión y resultados académicos "no siempre responde de manera lineal a la máxima 'a más dinero, mejores resultados'“ (Carpio, 2013, párr. 11), el dinero es importante, pero constituye solo el $28 \%$ de las diferencias de desempeño. Hay muchos países pobres a los que les va bien y muchos países ricos a los que les va mal. (Miranda y Schleicher, 2009, p. 24).

\section{Equipamiento e infraestructura adecuados}

Que responda pedagógicamente a las necesidades que se van presentando. 


\section{Maestros de calidad}

Uno de los puntos comunes de los países que lideran PISA es la alta prioridad dada a los docentes en: formación, profesionalismo, respeto social y remuneración (Ibídem, p. 26). Los países destacados ponen énfasis en la selección de maestros, los incentivan a trabajar juntos y facilitan su autonomía (Agencia EFE, 2013, párr. 5). Los maestros son seleccionados del primer tercio de cada promoción de graduados: el primer $5 \%$ en Corea del Sur, el $10 \%$ superior en Finlandia y el $30 \%$ superior en Singapur y Hong Kong. Barber y Mourshed (2008, p. 15). Para que una persona pueda convertirse en un docente eficiente debe poseer: alto nivel general de lengua y aritmética, fuertes capacidades interpersonales y de comunicación, deseo de aprender y motivación para enseñar.

\section{Incremento de horas en asignaturas básicas}

La OCDE de las 907 horas académicas que emplea de manera general, destina 145 para matemáticas, 143 para lengua y 133 para ciencias. (Carpio, 2013, párr. 16).

\section{Diversificación pertinente del Diseño Curricular Nacional (DCN)}

Respetando su enfoque y relacionándolo con las competencias en las que se basa la evaluación PISA (Miranda y Schleicher, 2009, p. 14) y dando una mirada al contexto cultural de la institución educativa. (Ibídem, p. 23).

\section{Gradualidad de contenidos}

Que sean apropiados para cada ciclo escolar.

\section{Textos escolares apropiados}

Con distintos tipos de texto, formatos continuos y discontinuos que propicien una lectura intertextual. Las lecturas deben aumentar el grado de complejidad y abstracción en su contenido, prácticas y ejercicios. (Flores y Meneses, 2013, párr. 17). 


\section{Aplicación de procesos cognitivos}

Por parte de los estudiantes, así adquirirán la competencia lectora.

\section{Desarrollo del hábito de lectura}

En los hogares de los estudiantes, pues este hábito es esencial para la comprensión (Ibídem).

\section{Acompañamiento personalizado}

Lograr un alto nivel en la comprensión lectora, a decir de Miranda y Schleicher (2009, p. 25) se consigue cuando alumnos y docentes interactúan con los textos para comprenderlos.

\section{Evaluación adecuada}

Evaluar con precisión las fortalezas y debilidades de cada alumno, para proceder correctamente (Barber y Mourshed, p. 28).

\section{Enfoque sociolingüístico para enseñar comunicación}

Pues este enfoque emplea la investigación - acción, logrando de ese modo superar las dificultades de aprendizaje que se van presentando (Maquera, 2013, p. 67).

\section{Lectura veloz}

Pues ello permite procesar de manera óptima textos extensos y complejos en el tiempo requerido en la evaluación (Flores y Meneses, párr. 26).

\section{Administración correcta del tiempo}

Para evitar el uso vano del tiempo en información vacía en las redes sociales (Ibídem, párr. 30) y asimismo propiciar el desarrollo del pensamiento crítico en la lectura virtual. 


\section{Expectativas de los padres}

Los escolares cuyos padres tienen expectativas altas para el futuro, rinden mejor en clase, ya que se sienten más seguros y motivados (Agencia EFE, 2013, párr. 2).

\section{Compromiso de estudiantes con su institución}

Los alumnos comprometidos hacen lo necesario para alcanzar sus objetivos académicos, logrando el éxito educativo (Carpio, 2013, párr. 19).

\section{Conclusiones}

- Los factores clave para elevar la calidad de la competencia lectora son: presupuesto conveniente, equipamiento e infraestructura adecuados, maestros de calidad, incremento de horas en asignaturas básicas, diversificación pertinente del DCN, gradualidad de contenidos, textos escolares apropiados, aplicación de procesos cognitivos, desarrollo del hábito de lectura, acompañamiento personalizado, evaluación adecuada, enfoque sociolingüístico para enseñar comunicación, lectura veloz, administración correcta del tiempo, expectativas de los padres y compromiso de los estudiantes con su institución.

- Como parte del sistema educativo adventista no debemos dejarnos atar ni siquiera por un hilo a la tendencia educativa de los que no disciernen la voz de Dios y no quieren escuchar sus mandamientos (White, 2008 a, p. 195). Por lo tanto, el modelo educativo que más se asemeja a la educación adventista y que se puede considerar es el de Finlandia, pues emplea la lectura de la Biblia para el desarrollo de los valores y la espiritualidad de su sociedad.

- Siempre es posible mejorar, incluso cuando el contexto sociocultural y económico no sea el más propicio, así lo sostiene Jakubowski (2011, p. 4) al señalar que la mejora entre los alumnos con menor competencia es posible en todos los países y es esencial para la reducción de las desigualdades educativas y la mejora de la competencia lectora. 
- La educación adventista que sigue a cabalidad las orientaciones de la Biblia y del Espíritu de Profecía, es la mejor opción, pues su formación trasciende esta vida hasta la eternidad. Los que consideran como esencial el conocimiento que se obtiene en la educación secular están cometiendo un grave error (White, 1923, p. 535).

\section{Belinda Vasti Maquera Chambi Universidad Peruana Unión email: belinda.maquera@upeu.pe}

Recibido: 18 de diciembre de 2013 Aceptado: 30 de enero de 2014

\section{Referencias}

Agencia EFE, Prueba PISA. (2013). ¿Cuál es la clave de los países con mejor puntuación? El Comercio.pe. Recuperado el 04 de diciembre de 2013. http://elcomercio.pe/actualidad/1668006/noticia-prueba-pisa-cual-clave-paises-mejor-puntuacion

Barber, M. \& Moursehd, M. (2008). Cómo hicieron los sistemas educativos con mejor desempeño del mundo para alcanzar sus objetivos. Programa de Promoción de la Reforma Educativa en América Latina y el Caribe. Chile: San Marino.

Cadwallader, E. (2010). Principios de la Educación Adventista en el Pensamiento de Elena de White. Filosofía, objetivos, métodos y misión. México: Editorial Universitaria Iberoamericana.

Carpio, J. (2013). El informe PISA recomienda mayor autonomía de los centros educativos y rendición de cuentas. Rtve.es. Recuperado el (03 de diciembre de 2013). http://www.rtve. es/noticias/20131203/informe-pisa-senala-mal-reparto-del-gasto-educativo-horaslectivas/809343.shtml

Flores, M. \& Meneses, M. (2013) Los resultados de la aplicación de prueba PISA en Perú: Desencuentros y oportunidades. Recuperado el marzo, 2013. http://victormazzihuaycucho. blogspot.com/2013/03/dossier-educacion-en-el-peru-problemas.html

Hernández, R. (2013). Finlandia: Un secreto a la vista de todos. Educación y Cultura AZ, 73,6-13, México.

Jakubowsky, M. (2011). Mejorar el rendimiento desde el nivel más bajo. In Focus, 2, 1-4. Recuperado de http://www.oecd.org/pisa/pisaproducts/pisainfocus/48363354.pdf

Maquera, B. (2013). Enseñanza y aprendizaje de la gramática a través de estrategias del enfoque sociolingüístico [Resumen]. Libro de resúmenes del I COSUDI y III CONACIN, 67, 152.

Miranda, L. \& Schleicher, A. (2009). La educación peruana en el contexto de PISA. Perú: Santillana S. A. 


\section{Belinda Maquera Chambi}

Organización para la Cooperación y el Desarrollo Económico (s/f). El Programa PISA de la OCDE.

Qué es y para qué sirve. España: Grupo Santillana.

Torres, R. (2013). ¿China, Corea del Sur o Finlandia? Educación y Cultura AZ, 73, 24 - 44, México.

Unidad de Medición de la Calidad Educativa (2013). PISA 2012. Recuperado de http://umc.minedu.gob.pe/?p=1694

Vilela, J. (2010) ¿Y cómo está la educación en Finlandia? Recuperado de http://blog.pucp.edu.pe/ item/48262/y-como-esta-la-educacion-en-finlandia

White, E. (s/f). La edificación del carácter y la formación de la personalidad. Argentina: Casa Editora Sudamericana.

White, E. (1923). Fundamentals of Christian Education. Setenta y cuatro artículos, ordenados cronológicamente desde 1872 hasta 1915.

White, E. (1957). Mensajes para los jóvenes. Argentina: Casa Editora Sudamericana.

White, E. (1958). La Educación. Argentina: Casa Editora Sudamericana.

White, E. (1974). Conducción del Niño. Argentina: Casa Editora Sudamericana.

White, E. (2008 a). Consejos para los maestros, padres y alumnos. Argentina: Casa Editora Sudamericana.

White, E. (2008 b). La educación cristiana. Argentina: Casa Editora Sudamericana. 